УДК 539.374

\title{
On Elastoplastic Torsion of a Rod with Multiply Connected Cross-Section
}

\author{
Sergey I. Senashov \\ Alexander V. Kondrin \\ Siberian State Aerospace University \\ Krasnoyarsky Rabochy, 31, Krasnoyarsk, 660014 \\ Russia \\ Olga N. Cherepanova* \\ Institute of Mathematics and Computer Science \\ Siberian Federal University \\ Svobodny, 79, Krasnoyarsk, 660041
}

Russia

Received 24.05.2015, received in revised form 14.06.2015, accepted 15.07.2015

The classical problem of torsion of a straight rod with convex contour of the cross-section is considered in the paper. The cross-section is multiply connected domain. It is assumed that the region of plastic deformation occupies the whole outer boundary. To solve the problem the conservation laws are used. In the case when the boundary is piecewise smooth the solution is found in explicit form. Computer programs that allow one to find the elastic-plastic boundary in a rod under torsion with any precision are developed. Examples of calculation of elastic-plastic boundaries from presented analytical formulas are given. The obtained results are in good agreement in comparison with known solutions and experimental data.

Keywords: conservation laws, exact solution, unknown boundary, torsion problem of straight rod, multiply connected cross-section.

DOI: 10.17516/1997-1397-2015-8-3-343-351

We consider a prismatic rod in the Cartesian coordinate system $O x y z$. The axis $O z$ is directed along the ruling of the rod (Fig. 1).

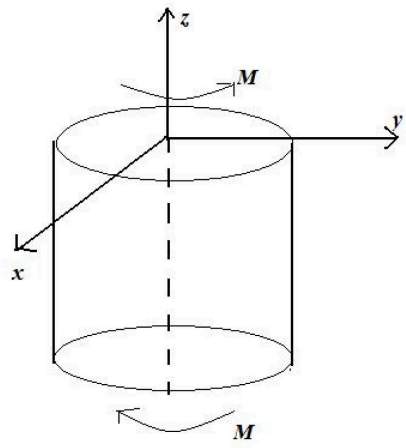

Fig. 1.

*cheronik@mail.ru

(c) Siberian Federal University. All rights reserved 
The rod is twisted around the axis $O z$ by couple of forces with the moment $M$. At some value of the moment $M$ part of the rod changes to plastic state. Plastic deformation begins at the outer contour $L_{0}$. The remaining part of the rod is in elastic state. Our problem is to define the boundary between elastic and plastic zones.

Statement of the problem. In the case of simply connected cross-section bounded by a piecewise smooth contour the problem was solved [1]. The computer program for rods of various cross sections was developed and registered. Some other rod torsion problems were considered $[2,3]$.

In the paper we assume that the cross-section of the rod is multiply connected domain. Fig. 2 shows the contour of the cross section of the rod in the plane $\operatorname{Oxy}\left(z=\right.$ const). Here $L_{0}$ is the outer contour and $L_{1}, L_{2}, L_{3}$ are internal contours.

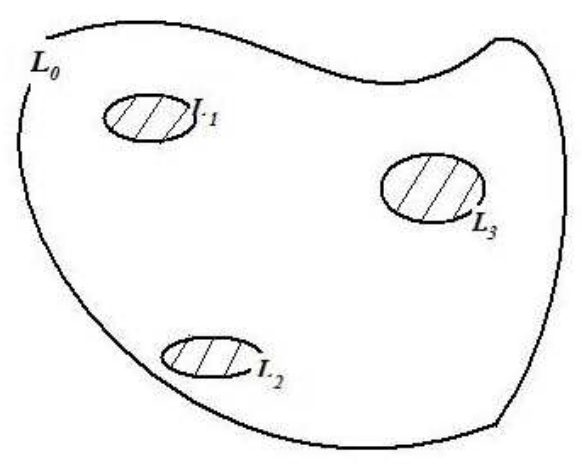

Fig. 2.

Further for simplicity we consider the elastic-plastic torsion of a rectangular rod. The crosssection is bounded by the outer contour $L_{0}$ and by internal contour $L_{1}$.

The plastic region $P$ is formed at the outer and inner contours at large value of the torque moment. We assume that the plastic region fully occupies these contours. Then plastic $P$ and elastic $E$ cross-section areas may occur and $L$ marks dividing lines between areas (Fig. 3 ).

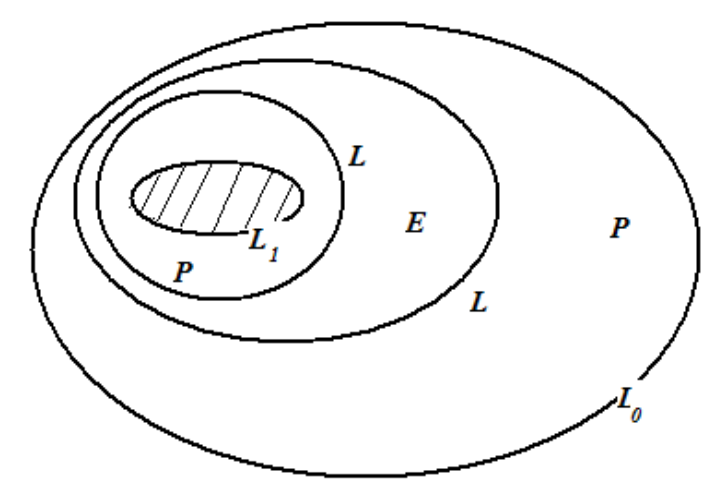

Fig. 3.

Solutions of the elastic-plastic stress problem in a rod were considered in many papers. Most of these solutions are based on some assumptions on the form of the boundary $L$ which is not 
known in advance. The original method to determine unknown boundary was proposed by B. D. Anin [4]. This method is based on the contact transformations and allows one to determine the boundary between elastic and plastic regions in rods of oval cross-section. Formulation of the problem and a detailed overview of the results can be found in [4] and in the references cited therein.

In the present work we use conservation laws to determine stress state for all internal points of the rod. We also provide analytical formulas for computing these stresses in the case of piecewise-smooth boundary of the cross section. Conservation laws have been successfully used to solve many problems in mathematics and mechanics. Summary of results and solved problems from various fields of mechanics can be found in [1-3,5-7].

Assume that $\tau_{x z}, \tau_{y z}$ are the nonzero components of the stress tensor. In the elastic zone they satisfy the equation of equilibrium

$$
\frac{\partial \tau_{x z}}{\partial x}+\frac{\partial \tau_{y z}}{\partial y}=0
$$

and equations

$$
\tau_{x z}=G \theta\left(\frac{\partial \psi}{\partial x}-y\right), \tau_{y z}=G \theta\left(\frac{\partial \psi}{\partial y}+x\right) .
$$

Here function $\theta \psi(x, y)$ defines warping of the cross section, $\theta$ is the twisting constant, $G$ is the modulus of elasticity which is constant for a given material.

We introduce the stress function $\varphi$ as follows

$$
\tau_{x z}=\frac{\partial \varphi}{\partial y}, \tau_{y z}=-\frac{\partial \varphi}{\partial x},
$$

then we obtain the following equation for function $\varphi$

$$
\frac{\partial^{2} \varphi}{\partial x^{2}}+\frac{\partial^{2} \varphi}{\partial y^{2}}=a
$$

where $a=-2 G \theta$ is the non-zero constant.

In the plastic region components $\tau_{x z}, \tau_{y z}$ satisfy both equation of equilibrium and the condition of plasticity

$$
\tau_{x z}^{2}+\tau_{y z}^{2}=1
$$

Here for simplicity of further calculations plasticity constant is assumed to be equal to one.

Substituting the stress function into equation (5) we have

$$
\left(\frac{\partial \varphi}{\partial x}\right)^{2}+\left(\frac{\partial \varphi}{\partial y}\right)^{2}=1
$$

Boundary conditions. We assume stress-free boundary conditions on the side surface. It means that $\frac{\partial \varphi}{\partial l}=0$ on the contours $L_{0}, L_{1}$. Here $\vec{l}=\left(l_{1}, l_{2}\right)$ is the tangent vector to the contours $L_{0}, L_{1}$. Then we obtain that $\varphi=$ const along contours $L_{0}, L_{1}$, and for each contour this constant may have different value. It is usually assumed that $\varphi=0$ on $L_{0}$.

Finally we obtain the following problem.

In the elastic region it is necessary to solve the equation

$$
\frac{\partial^{2} \varphi}{\partial x^{2}}+\frac{\partial^{2} \varphi}{\partial y^{2}}=a
$$


in the region of plasticity the function $\varphi$ satisfies the equation

$$
\left(\frac{\partial \varphi}{\partial x}\right)^{2}+\left(\frac{\partial \varphi}{\partial y}\right)^{2}=1
$$

on the contours $L_{0}, L_{1}$ we have the following conditions for function $\varphi$

$$
\frac{\partial \varphi}{\partial l}=0 \quad \text { or } \quad \frac{\partial \varphi}{\partial l_{1}}+\frac{\partial \varphi}{\partial l_{2}}=0,
$$

on the boundary $L$ function $\varphi$ is continuous. It is necessary to find $\varphi$ in elastic and plastic regions and also one needs to find surface $L$. We introduce the following notation $\varphi_{x}^{\prime}=u, \varphi_{y}^{\prime}=v$. Then equations (7)-(8) take the form

$$
\begin{gathered}
F_{1}=u_{x}^{\prime}+v_{y}^{\prime}-a=0 \\
u^{2}+v^{2}=1 .
\end{gathered}
$$

We also have the equality

$$
F_{2}=u_{y}^{\prime}-v_{x}^{\prime}=0
$$

Definition. Vector $(A, B)$ is conserved current for the system of equations (10), (12) if the following relation is satisfied

$$
\partial_{x} A+\partial_{y} B=\Delta_{1} F_{1}+\Delta_{2} F_{2}=0
$$

Here $\Delta_{1}, \Delta_{2}$ are some linear differential operators.

It means that functions $A$ and $B$ satisfy the following conservation law

$$
\partial_{x} A+\partial_{y} B=0
$$

for all solutions of system (10), (12).

The conservation law (14) in virtue of equations (9), (11) has the form

$$
A_{x}+A_{u} u_{x}+A_{v} v_{x}+B_{y}+B_{u} u_{y}+B_{v} v_{y}=0
$$

or taking into account that $u_{x}=a-v_{y}$ and $u_{y}=v_{x}$,

$$
A_{x}+A_{u} a-A_{u} v_{y}+A_{v} v_{x}+B_{y}+B_{u} v_{x}+B_{v} v_{y}=0 .
$$

It follows from the last equality that functions $A$ and $B$ satisfy the following equations

$$
\begin{gathered}
A_{x}+A_{u} \cdot a+B_{y}=0, \\
B_{v}-A_{u}=0, A_{v}+B_{u}=0 .
\end{gathered}
$$

Note that equations (16) are the Cauchy-Riemann equations.

Let us consider domain $D$ bounded by the contours $L_{0}, L_{1}$, . We assume that the area of plasticity $P$ completely encloses the elastic area $E$. It is also assumed that $L_{0}, L_{1}$ are smooth oriented curves, i.e., continuously differentiable curves without singular points. The boundary formed by the contours $L_{0}$ and $L_{1}$ is denoted by $\Gamma$.

It follows from conservation law (14) that

$$
\iint_{D}\left(\partial_{x} A+\partial_{y} B\right) d x d y=0 .
$$


Using Green's formula, we obtain from (17) the following relation

$$
\oint_{\Gamma} A d y-B d x=0 .
$$

Our problem is to find a domain $E$ that belongs to domain $D$ together with the boundary $L$ in which the inequality $u^{2}+v^{2}<1$ is true. Let us assume that $A=\alpha u+\beta v$ and $B=\alpha v-\beta u+\gamma$ then

$$
\begin{gathered}
A_{x}=\alpha_{x} u+\alpha u_{x}+\beta_{x} v+\beta v_{x}, \\
B_{y}=\alpha_{y} v+\alpha v_{y}-\beta_{y} u-\beta u_{y}+\gamma_{y},
\end{gathered}
$$

where $\alpha, \beta, \gamma$ are unknown functions that depend only on $x, y$.

According to conservation law (14), we obtain the equality

$$
A_{x}+B_{y}=\alpha_{x} u+\alpha u_{x}+\beta_{x} v+\beta v_{x}+\alpha_{y} v+\alpha v_{y}-\beta_{y} u-\beta u_{y}+\gamma_{x}=0,
$$

from which the conditions for the functions $\alpha, \beta$ and $\gamma$ follow

$$
\left\{\begin{array}{l}
\alpha_{x}-\beta_{y}=0 \\
\beta_{x}+\alpha_{y}=0 \\
a \alpha+\gamma_{y}=0
\end{array}\right.
$$

Let us consider two solutions of system (22). The first solution has the form

$$
\alpha_{1}=\frac{x-x_{0}}{\left(x-x_{0}\right)^{2}+\left(y-y_{0}\right)^{2}}, \beta_{1}=-\frac{y-y_{0}}{\left(x-x_{0}\right)^{2}+\left(y-y_{0}\right)^{2}}, \gamma_{1 y}=-a \frac{x-x_{0}}{\left(x-x_{0}\right)^{2}+\left(y-y_{0}\right)^{2}},
$$

then

$$
\gamma_{1}=-a \cdot \operatorname{arctg} \frac{y-y_{0}}{x-x_{0}}
$$

The second solution takes the form

$$
\alpha_{2}=\frac{y-y_{0}}{\left(x-x_{0}\right)^{2}+\left(y-y_{0}\right)^{2}}, \beta_{2}=\frac{x-x_{0}}{\left(x-x_{0}\right)^{2}+\left(y-y_{0}\right)^{2}}, \gamma_{2 x}=-a \frac{x-x_{0}}{\left(x-x_{0}\right)^{2}+\left(y-y_{0}\right)^{2}} .
$$

Then

$$
\gamma_{2}=-\frac{a}{2} \cdot \ln \left(\left(x-x_{0}\right)^{2}+\left(y-y_{0}\right)^{2}\right) .
$$

Let us rewrite equation (18) for functions $A$ and $B$

$$
\begin{gathered}
\oint_{\Gamma} A d y-B d x=\oint_{\Gamma}(\alpha u+\beta v) d y-(\alpha v-\beta u+\gamma) d x=\oint_{\Gamma}\left(-\alpha \frac{l_{2}}{l_{1}}+\beta\right) v d y- \\
-\left(\alpha \frac{l_{1}}{l_{2}}-\beta\right) u d x-\oint_{\Gamma} \gamma d x=\oint_{\Gamma}\left(-\alpha \frac{l_{2}}{l_{1}}+\beta\right)-\left(\alpha \frac{l_{1}}{l_{2}}-\beta\right) \frac{\partial \varphi}{\partial x} d x-\gamma d x= \\
=\oint_{\Gamma}\left(-\alpha \frac{l_{2}}{l_{1}}\right) v d y-\left(\alpha \frac{l_{1}}{l_{2}}\right) d x-\oint_{\Gamma} \gamma d x+\oint_{\Gamma} \beta\left(\frac{\partial \varphi}{\partial y} d y+\frac{\partial \varphi}{\partial x} d x\right)= \\
=\oint_{\Gamma} \alpha u d y-(\alpha v+\gamma) d x=0 .
\end{gathered}
$$

Let us divide the boundary Gamma into seven parts, i.e., $\Gamma=\Gamma_{1}+\Gamma_{2}+\Gamma_{3}+\Gamma_{4}+\Gamma_{5}+\Gamma_{6}+\Gamma_{7}$, where $\Gamma_{3}$ is the circle $\left(x-x_{0}\right)^{2}+\left(y-y_{0}\right)^{2}=R^{2}, \Gamma_{1}=L_{0}$ and $\Gamma_{5}=L_{1}$ (Fig. 4). Then relation (26) becomes

$$
\oint_{\Gamma} A d y-B d x=\oint_{\Gamma} \alpha u d y-(\alpha v+\gamma) d x=\sum_{i=1}^{7} \oint_{\Gamma_{i}}(\alpha u d y-(\alpha v+\gamma) d x)=0 .
$$




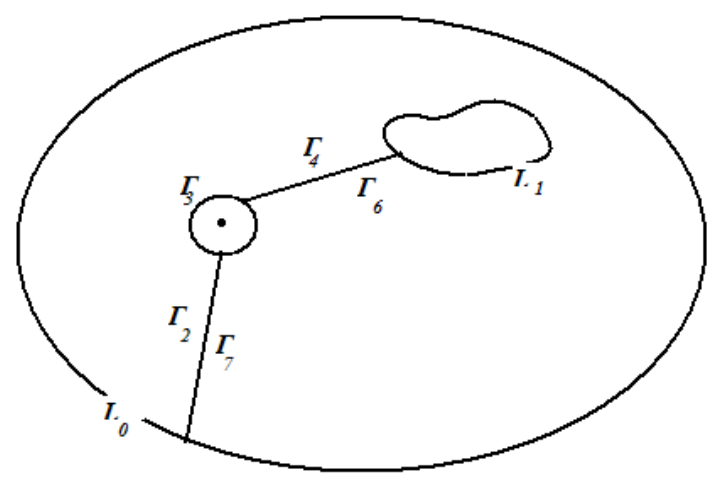

Fig. 4.

It is obvious that

$$
\oint_{\Gamma_{2}} \alpha u d y-(\alpha v+\gamma) d x+\oint_{\Gamma_{7}} \alpha u d y-(\alpha v+\gamma) d x=0
$$

and

$$
\oint_{\Gamma_{4}} \alpha u d y-(\alpha v+\gamma) d x+\oint_{\Gamma_{6}} \alpha u d y-(\alpha v+\gamma) d x=0 .
$$

Taking into account this condition, equation (25) takes the form

$$
\oint_{\Gamma_{3}} \alpha u d y-(\alpha v+\gamma) d x=-\oint_{\Gamma_{1}} \alpha u d y-(\alpha v+\gamma) d x+\oint_{\Gamma_{5}} \alpha u d y-(\alpha v+\gamma) d x .
$$

Let us calculate the integral $\oint_{\Gamma_{1}}$ where $\Gamma_{1}$ is the circle of radius $R$. We introduce

$$
\alpha=\alpha_{1}=\frac{x-x_{0}}{\left(x-x_{0}\right)^{2}+\left(y-y_{0}\right)^{2}}, \quad \beta=\beta_{1}=\frac{y-y_{0}}{\left(x-x_{0}\right)^{2}+\left(y-y_{0}\right)^{2}}, \quad \gamma_{1}=-a \cdot \operatorname{arctg} \frac{y-y_{0}}{x-x_{0}}
$$

We use the polar coordinate system

$$
\left\{\begin{array}{l}
x-x_{0}=R \cos \varphi \\
y-y_{0}=R \sin \varphi
\end{array}\right.
$$

Then

$$
\left\{\begin{array}{l}
d x=-R \sin \varphi d \varphi, \\
d y=R \cos \varphi d \varphi,
\end{array} \quad \alpha=\frac{\cos \varphi}{R}, \quad \beta=\frac{\sin \varphi}{R}, \gamma=-\alpha \varphi .\right.
$$

As a result at $R \rightarrow 0$ we have

$$
\oint_{\Gamma_{1}} \alpha u d y-(\alpha v+\gamma) d x=\pi u\left(x_{0}, y_{0}\right)
$$

Similarly when $\alpha=\alpha_{2}, \beta=\beta_{2}, \gamma=\gamma_{2}$ we have

$$
\oint_{\Gamma_{1}} \alpha u d y-(\alpha v+\gamma) d x=\pi v\left(x_{0}, y_{0}\right) .
$$


As a result from equation (14) follows

$$
\begin{aligned}
& \oint_{\Gamma_{3}} \alpha_{1} u d y-\left(\alpha_{1} v+\gamma_{1}\right) d x=\pi u\left(x_{0}, y_{0}\right), \\
& \oint_{\Gamma_{3}} \alpha_{2} u d y-\left(\alpha_{2} v+\gamma_{2}\right) d x=\pi v\left(x_{0}, y_{0}\right) .
\end{aligned}
$$

The curve $\Gamma_{1}=L_{0}$ is represented in parametric form

$$
L_{0}: x=f_{0}(t), y=\varphi_{0}(t), \quad 0 \leqslant t \leqslant T_{0} .
$$

The curve $\Gamma_{5}=L_{1}$ has the following parametric form

$$
L_{1}: x=f_{1}(t), y=\varphi_{1}(t), \quad 0 \leqslant t \leqslant T_{1} .
$$

Functions $f^{\prime}(t)$ and $\varphi^{\prime}(t)$ are derivatives of functions $f(t)$ and $\varphi(t)$, respectively.

Taking into account (27), functions $u\left(x_{0}, y_{0}\right)$ and $v\left(x_{0}, y_{0}\right)$ from (34), (35) take the form

$$
\begin{aligned}
u\left(x_{0}, y_{0}\right) & =\frac{1}{\pi}\left[\int_{0}^{T_{0}}\left(\frac{\left(f(t)-x_{0}\right) \sqrt{\left(f^{\prime}(t)\right)^{2}+\left(\varphi^{\prime}(t)\right)^{2}}}{\sqrt{\left(f(t)-x_{0}\right)^{2}+(\varphi(t)-y-0)^{2}}}+\operatorname{af}(t) \operatorname{arctg} \frac{\varphi(t)-y_{0}}{f(t)-x_{0}}\right) d t-\right. \\
& \left.-\int_{0}^{T_{1}}\left(\frac{\left(f(t)-x_{0}\right) \sqrt{\left(f^{\prime}(t)\right)^{2}+\left(\varphi^{\prime}(t)\right)^{2}}}{\sqrt{\left(f(t)-x_{0}\right)^{2}+(\varphi(t)-y-0)^{2}}}\right) d t\right] \\
v\left(x_{0}, y_{0}\right)= & \frac{1}{\pi}\left[\int _ { 0 } ^ { T _ { 0 } } \left(\frac{\left(\varphi(t)-y_{0}\right) \sqrt{\left(f^{\prime}(t)\right)^{2}+\left(\varphi^{\prime}(t)\right)^{2}}}{\sqrt{\left(f(t)-x_{0}\right)^{2}+\left(\varphi(t)-y_{0}\right)^{2}}}+\frac{a}{2} f^{\prime}(t) \ln \left(\left(f(t)-x_{0}\right)^{2}+\right.\right.\right. \\
& \left.\left.+\left(\varphi(t)-y_{0}\right)^{2}\right) d t-\int_{0}^{T_{1}}\left(\frac{\left(\varphi(t)-y_{0}\right) \sqrt{\left(f^{\prime}(t)\right)^{2}+\left(\varphi^{\prime}(t)\right)^{2}}}{\sqrt{\left(f(t)-x_{0}\right)^{2}+(\varphi(t)-y-0)^{2}}}\right) d t\right]
\end{aligned}
$$

Now we can compute the value of

$$
u^{2}+v^{2}
$$

at the point $\left(x_{0}, y_{0}\right)$. Point belongs to the plastic region if the value of $(39)$ is greater or equal to the unit. Point belongs to the elastic region if the value of (39) is less than unity.

Computer programs that allow one to find the plastic and elastic regions in a twisted rod with any precision have been developed. They are based on relations (4), (35).

The application of the proposed method to test problems shows good agreement with the known solutions (Figs. 5, 6).

The work was supported by the Ministry of education and science grant B 180-14.

The work was supported by the Russian Federation Government grant to conduct scientific research under the guidance of leading scientists in the Siberian Federal University, contract no. 14. Y 26.31.0006.

\section{References}

[1] S.I.Senashov, O.N Cherepanova, A.V.Kondrin, On elastoplastic torsion rod, Vestnik SibGAU, 49(2013), no. 3, 100-103 (in Russion).

[2] S.I.Senashov, O.N.Cherepanova, A.V Kondrin, E.V.Filushina, The calculation of the stress state in the interior of elastic-plastic rod of constant cross section, Certificate of state registration of the computer 013618484 . 


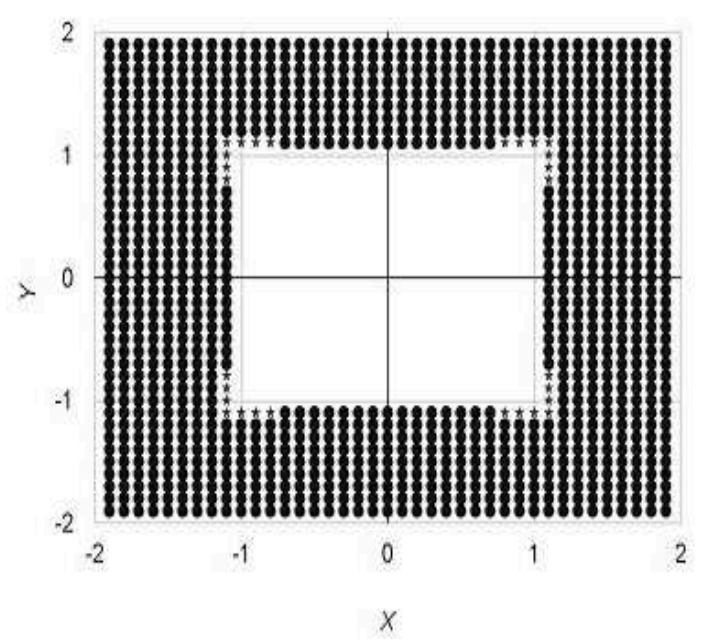

The parameter characterizing the torsion -02

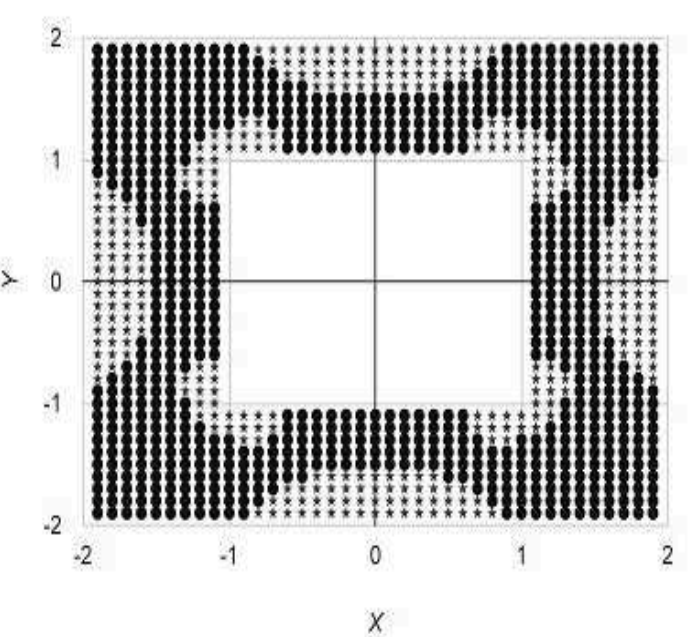

The parameter characterizing the torsion -05

Fig. 5. Calculation for a rectangular hole

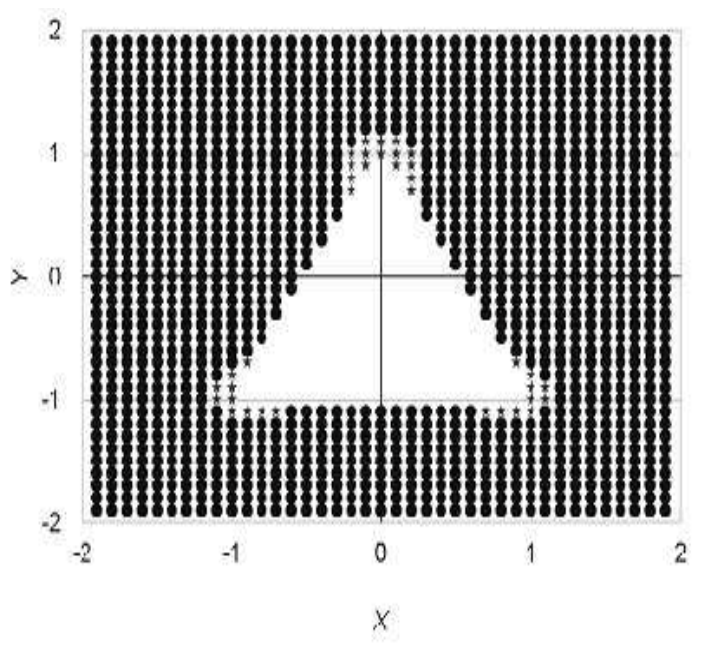

The parameter characterizing the torsion -01

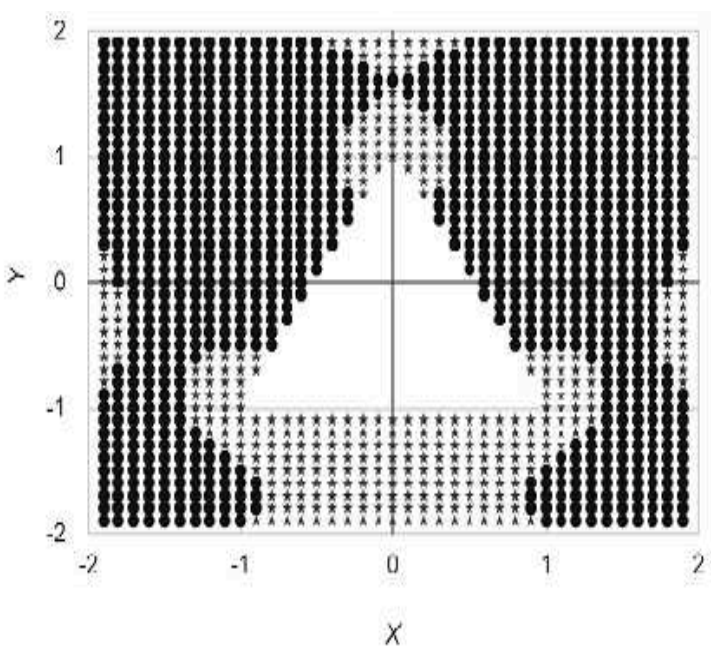

The parameter characterizing the torsion -05

Fig. 6. Calculation for a triangular hole

[3] S.I.Senashov, O.N.Cherepanova, A.V.Kondrin, A.N.Yahno, E.V.Filushina, Building elastoplastic boundary problems arising in torsion rod with a rectangular cross section of rectangular shape, Certificate of state registration of the computer 20146616472.

[4] Б.Д.Anin, G.P.Cherepanov, Elastic-plastic problem, Novosibirsk, Nauka, 1983 (in Russion).

[5] P.P.Kiryakov, S.I.Senashov, A.N.Yahno, Application symmetries and conservation laws to solving differential equations, Izdatelstvo SO RAN, Novosibirsk, 2001 (in Russion).

[6] S.I.Senashov, A.N.Yachno, Conservation Laws, Hodograph Transformaition and Boundary Value Problems of Plane Plasticity, SIGMA, 8(2012), 071. 
[7] S.I.Senashov Conservation laws in the problem of the longitudinal plane wave loads in elasticplastic rod, Vestnik SibGAU, 36(2011), no, 3, 82-85 (in Russion).

\title{
Об упругопластическом кручении стержня с многосвязным поперечным сечением
}

\section{Сергей И. Сенашов \\ Александр В. Кондрин \\ Ольга Н. Черепанова}

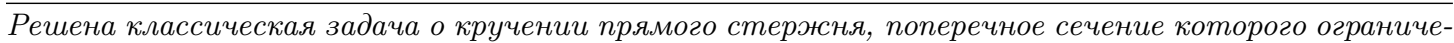
но выпуклым контуром. Поперечное сечение является многосвязной областъю. Предполагается, что пластическая область охватывает всю внешнюю граничу. Для решения задачи используются законы сохранения. В случае, когда гранича кусочно-гладкая, решение найдено квадратурой. Написаны программы, позволяющие с любой точностью строить упруго-пластическую граничу в скручиваемом стержне. Приведены примеры вычисления упруго-пластических границ, на ЭВМ по найденным аналитическим формулам. Сравнение с известными решениями и экспериментальными данными показало хорошее совпадение результатов.
\end{abstract}

Ключевые слова: законы сохранения, точные решения, неизвестная граница, задача кручения прямого стержня, многосвязное сечение. 